Algebras and Representation Theory manuscript No.

(will be inserted by the editor)

\title{
Cohomology of block ideals of finite group algebras and stable elements
}

\author{
Hiroki Sasaki
}

Received: date / Accepted: date

\begin{abstract}
We shall show that an element in the cohomology ring of a defect group of a block ideal of the group algebra over an algebraically closed filed of prime characteristic belongs to the cohomology ring of the block ideal if and only if it's embedding into the Hochschild cohomology ring of the group algebra of the defect group is stable with respect to the source algebra of the block ideal.
\end{abstract}

Keywords finite group $\cdot$ block ideal $\cdot$ Brauer correspondence $\cdot$ Hochschild cohomology $\cdot$ group cohomology $\cdot$ stable elements

Mathematics Subject Classification (2000) 20 C20 20 J06

\section{Introduction}

Let $G$ be a finite group and let $k$ be an algebraically closed field of prime characteristic $p>0$ dividing the order of $G$. Linckelmann [5] defined the cohomology ring of a block ideal of $k G$.

Let $B$ be a block ideal of $k G$ with a defect group $D$. Let $X$ be a source module of $B$, namely an indecomposable direct summand of $B$ as $k\left[G \times D^{\text {op }}\right]$-module having $\triangle D$ as vertex; $X$ has a trivial source. The source module $X$ can be written as $X=k G i$ with a source idempotent $i$; let $\gamma$ be the point containing $i$. Let $\left(D, b_{D}\right)$ be the Sylow $B$-subpair such that the Brauer construction $X(D)$ belongs to $b_{D}$.

Definition 1 Under the notation above, the cohomology ring $H^{*}(G, B ; X)$, which is also denoted by $H^{*}\left(G, B ; D_{\gamma}\right)$, is defined to be the subset of the cohomology ring $H^{*}(D, k)$ consisting of the elements $\zeta$ satisfying the following stability condition:

$$
\operatorname{res}_{Q}{ }^{g} \zeta=\operatorname{res}_{Q} \zeta
$$

This work was supported by Japan Society for the Promotion of Science Grant-in-Aid for Scientific Research (C) (22540013)

Shinshu University, School of General Education

Matsumoto, 390-8621, Japan

E-mail: sasakitk@ shinshu-u.ac.jp 
for all $B$-subpair $\left(Q, b_{Q}\right) \leqslant\left(D, b_{D}\right)$ and all element $g \in N_{G}\left(Q, b_{Q}\right)$.

One of the main theorems in [5] is that every element $\zeta \in H^{*}(G, B ; X)$ is embedded, via diagonal embedding, into the subring of the Hochschild cohomology ring $H H^{*}(k D)$ of the group algebra $k D$ which is stable with respect to $i k G i=X^{*} \otimes_{B} X$. Here $i k G i$ is the source algebra of the block $B$, being viewed as a $(k D, k D)$-bimodule. We shall in the next section recall the definition of stable elements in Hochschild cohomology rings.

If $B_{0}$ is the principal block of $k G$, then, $P$ being a Sylow $p$-subgroup of $G$, the block cohomology $H^{*}\left(G, B_{0}\right) \subset H^{*}(P, k)$ coincides with the subset consisting of the elements $\zeta$ satisfying the stability condition:

$$
\operatorname{res}_{P \cap g P} g_{\zeta}=\operatorname{res}_{P \cap 8 P} \zeta
$$

for all $g \in G$; such $\zeta$ s are said to be $G$-stable.

The stable elements theorem says that an element $\zeta \in H^{*}(P, k)$ is $G$-stable if and only if it belongs to the image of the restriction map $\operatorname{res}_{P}: H^{*}(G, k) \rightarrow H^{*}(P, k)$. Namely the block cohomology of the principal block is isomorphic to the usual cohomology ring $H^{*}(G, k)$ via restriction map from $G$ to $P$. An element $\zeta$ in $H^{*}(P, k)$ is $G$-stable if and only if its diagonal embedding in $H H^{*}(k P)$ is $k G$-stable, where $k G$ is considered as a $(k P, k P)$-bimodule. Thus we can say, identifying $H^{*}(G, k)$ with the image $\operatorname{Im} \operatorname{res}_{P}$, that an element $\zeta$ in $H^{*}(P, k)$ belongs to $H^{*}(G, k)$ if and only if its diagonal embedding in $H H^{*}(k P)$ is $k G$-stable. Hence, if we expect the block version of this fact, then we could say that the theorem by Linckelmann is the "only if" part. Our purpose of this note is to show that the "if" part does hold. We let $\delta_{D}: H^{*}(D, k) \rightarrow H H^{*}(k D)$ denote the diagonal embedding.

Theorem 1 An element $\zeta \in H^{*}(D, k)$ belongs to $H^{*}(G, B ; X)$ if and only if the diagonal embedding $\delta_{D} \zeta \in H H^{*}(k D)$ is ikGi-stable.

Using the theorem above, we shall examine a relationship between cohomology rings of blocks which are in Brauer correspondence. Let $H$ be a subgroup of $G$ containing $D C_{G}(D)$; let $C$ be a Brauer correspondent of $B$ in $k H$ with $D$ as a defect group. It is worthy to investigate whether the cohomology ring of $B$ is contained in that of $C$. To analyze this situation we have to take source modules $X$ of $B$ and $Y$ of $C$ in order that these are in Green correspondence with respect to $\left(G \times D^{\mathrm{op}}, \Delta D, H \times D^{\mathrm{op}}\right)$. Take the Green correspondent $L=L(B, C)$ of $C$, being viewed as an indecomposable $k\left[H \times H^{\mathrm{op}}\right]$-module, with respect to $\left(G \times H^{\mathrm{op}}, \Delta D, H \times H^{\mathrm{op}}\right)$. Then we have

Theorem 2 Under the notation above the cohomology ring $H^{*}(G, B ; X)$ is contained in $H^{*}(H, C ; Y)$ if and only if $H^{*}(G, B ; X)$ is embedded, by the diagonal embedding, into the $X^{*} \otimes_{B} L \otimes_{C} Y$-stable subring of the Hochschild cohomology ring $H H^{*}(k D)$ of the group algebra $k D$. When the condition does hold, we have the following commutative diagram:

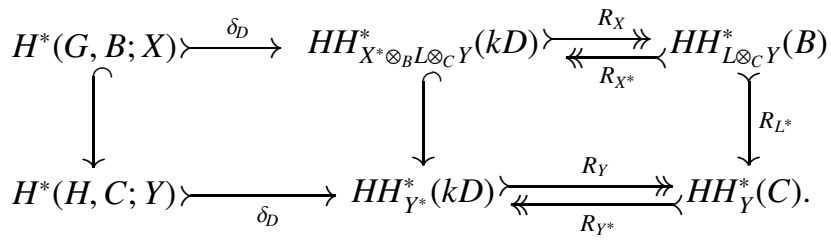


Here, for $M$ a bimodule, the map $R_{M}$ is the normalized transfer map defined by $M$ restricted to stable subrings of Hochschild cohomology rings. Theorems 1 and 2 will be proved in Section 2.

Broto, Levi and Oliver [1] shows that there exists a $(D, D)$-biset $S$ that induces a linear map $t_{S}: H^{*}(D, k) \rightarrow H^{*}(D, k)$ whose image is just the block cohomology $H^{*}(G, B ; X)$; the biset $S$ is called a characteristic biset. Taking Theorem 1 into account, we hope that such a characteristic biset must be taken very closely to the source algebra $i k G i$.

The Mackey double coset formula, $P$ being a Sylow $p$-subgroup, gives rise to

$$
\operatorname{Im~res}_{P}=\operatorname{Im}\left[t: H^{*}(P, k) \rightarrow H^{*}(P, k) ; \zeta \mapsto \sum_{G=\bigcup P g P} \operatorname{tr}^{P} \operatorname{res}_{P \cap g P} g_{\zeta}\right] .
$$

What should be the block cohomology version of this fact? Let

$$
i k G i \simeq \bigoplus_{D x D} k[D x D]
$$

be a decomposition into the direct sum of indecomposable $(k D, k D)$-bimodules; define a linear map as follows:

$$
t: H^{*}(D, k) \rightarrow H^{*}(D, k) ; \zeta \mapsto \sum_{D x D} \operatorname{tr}^{D} \operatorname{res}_{D \cap{ }^{x} D}{ }^{x} \zeta
$$

Then we would like to propose the following conjecture.

Conjecture Under the notation above it would follow that

$$
H^{*}(G, B ; X)=t H^{*}(D, k)
$$

Notice that the linear map $t: H^{*}(D, k) \rightarrow H^{*}(D, k)$ above is the restriction of the transfer map $t_{i k G i}: H H^{*}(k D) \rightarrow H H^{*}(k D)$ defined by the $(k D, k D)$-bimodule $i k G i$ via diagonal embedding:

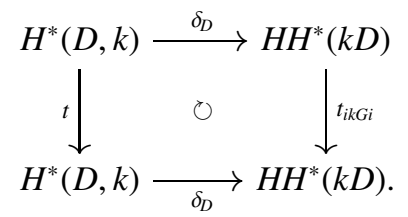

Remark 1 We see that $H^{*}(G, B ; X) \subseteq t H^{*}(D, k)$, as we have for $\zeta \in H^{*}(G, B ; X)$ that $\delta_{D} t(\zeta)=t_{i k G i} \delta_{D} \zeta=\pi_{i k G i} \delta_{D} \zeta$ and the relative projective element $\pi_{i k G i}$ is in fact a nonzero scalar.

In Section 3 we shall give a few conditions equivalent to Conjecture and examples.

Here we fix a symbol; if a module $M$ is isomorphic with a direct summand of a module $N$ then we write $M \mid N$. 


\section{Cohomology rings of block ideals-proofs of Theorems}

Before proceeding to the proofs of theorems we state some facts on stable elements in Hochschild cohomology rings of symmetric algebras.

Let $R$ be a commutative ring and let $A$ and $B$ be symmetric $R$-algebras.

Let $X$ be an $(A, B)$-bimodule such that as a left $A$-module $X$ is finitely generated and projective and as a right $B$-module $X$ is finitely generated and projective.

Definition 2 A pair $(\zeta, \theta) \in H H^{*}(A) \oplus H H^{*}(B)$ is called an $X$-stable pair if the elements $\zeta \otimes \operatorname{Id}_{X}$ and $\operatorname{Id}_{X} \otimes \theta$ in $\operatorname{Ext}_{A \otimes B^{\text {op }}}^{*}(X, X)$ coincide; we also say that $(\zeta, \theta)$ is $X$-stable. The element $\zeta \in H H^{*}(A)$ is said to be $X$-stable. The $X$-stable elements in $H H^{*}(A)$ form a subring, which is called the $X$-stable subring and is denoted by $H H_{X}^{*}(A)$.

If $(\zeta, \theta) \in H H^{*}(A) \oplus H H^{*}(B)$ is $X$-stable then $(\theta, \zeta) \in H H^{*}(B) \oplus H H^{*}(A)$ is $X^{*}$ stable, where $X^{*}$ is the $R$-dual of $X$.

Linckelmann [5, Corollary 3.8] says that if the relatively projective element $\pi_{X^{*}} \in$ $Z(B)$ is invertible then an $X \otimes_{B} X^{*}$-stable element in $H H^{*}(A)$ is also $X$-stable. The following is the converse to this fact, which is merely a restatement of Kawai and Sasaki [2, Lemma 2.1].

Proposition 3 The following hold.

1. If $\zeta \in H H^{n}(A)$ is $X$-stable then the pair $(\zeta, \zeta) \in H H^{n}(A) \oplus H H^{n}(A)$ is $X \otimes_{B} X^{*}$ stable. In particular we have $H H_{X}^{n}(A) \subset H H_{X \otimes_{B} X^{*}}^{n}(A)$.

2. Suppose that the relatively projective element $\pi_{X^{*}} \in Z(B)$ is invertible. Then we have $H H_{X}^{*}(A)=H H_{X \otimes_{B} X^{*}}^{*}(A)$; if $\zeta \in H H^{*}(A)$ is $X \otimes_{B} X^{*}$-stable then $(\zeta, \zeta) \in$ $H H^{n}(A) \oplus H H^{n}(A)$ is $X \otimes_{B} X^{*}$-stable.

Proof of Theorem 1 The "only if" part is [5, Theorem 5.6 (ii)]. We shall prove the "if" part. Let $\zeta \in H^{*}(D, k)$. We assume that $\delta_{D} \zeta \in H H^{*}(k D)$ is stable with respect to $i k G i=X^{*} \otimes_{B} X$.

We would like to show that $\zeta$ belongs to $H^{*}(G, B ; X)$.

Since the relatively projective element $\pi_{X} \in Z(B)$ is invertible, we see from Proposition 3 that $\left(\delta_{D} \zeta, \delta_{D} \zeta\right) \in H H^{*}(k D) \oplus H H^{*}(k D)$ is $i k G i$-stable. We see for a direct summand $k[D x D]$ of $i k G i$ that $\left(\delta_{D} \zeta, \delta_{D} \zeta\right)$ is $k[D x D]$-stable; Linckelmann [5, Lemma 5.3] implies that

$$
\operatorname{res}_{D \cap x_{D}}{ }^{x} \zeta=\operatorname{res}_{D \cap x} \zeta .
$$

Now, it is enough to show that the stability condition (S) holds for subpairs $\left(Q, b_{Q}\right)$ belonging to a conjugation family $\mathscr{F} \subseteq\left\{\left(Q, b_{Q}\right) \mid\left(Q, b_{Q}\right) \leqslant\left(D, b_{D}\right)\right\}$; the family $\mathscr{F}=\left\{\left(Q, b_{Q}\right) \mid\left(Q, b_{Q}\right) \leqslant\left(D, b_{D}\right)\right.$ is extremal $\}$ is a conjugation family. If $\left(Q, b_{Q}\right) \leqslant\left(D, b_{D}\right)$ is extremal, then $Q C_{D}(Q)$ is a defect group of $b_{Q}$.

Take a $B$-subpair $\left(Q, b_{Q}\right) \leqslant\left(D, b_{D}\right)$ which is extremal. Let $g \in N_{G}\left(Q, b_{Q}\right)$. Linckelmann [4, Lemma $3.3(\mathrm{v})]$ says that the $(k Q, k Q)$-bimodule $k[g Q]$ is isomorphic to a direct summand of $i k G i$ as $(k Q, k Q)$-bimodule.

Choose an indecomposable direct summand $k[D x D]$ of $i k G i$ such that $k[g Q]$ is a direct summand of $k[D x D]$ as $(k Q, k Q)$-bimodule:

$$
k[g Q] \mid k[D x D] .
$$


The $k\left[Q \times Q^{\text {op }}\right]$-module $k[g Q]$ has a trivial source with vertex ${ }^{(g, 1)} \Delta Q$ :

$$
k[g Q]=k\left[Q \times Q^{\mathrm{op}}\right] \otimes_{k[(, 1) \Delta Q]} k .
$$

The $k\left[D \times D^{\mathrm{op}}\right]$-module $k[D x D]$ has a trivial source with vertex ${ }^{(x, 1)} \Delta\left({ }^{x^{-1}} D \cap D\right)$ :

$$
k[D x D]=k\left[D \times D^{\mathrm{op}}\right] \otimes_{k\left[(x, 1) \Delta\left(x^{-1} D \cap D\right)\right]} k .
$$

Therefore, we have

$$
\left.k\left[Q \times Q^{\mathrm{op}}\right] \otimes_{k[(,, 1)} \Delta Q\right] k \mid{ }_{k\left[Q \times Q^{\mathrm{op}]}\right]} k\left[D \times D^{\mathrm{op}}\right] \otimes_{k\left[(x, 1) \Delta\left(x^{-1} D \cap D\right)\right]} k .
$$

Mackey decomposition theorem says that there exists an element $(a, b) \in D \times D^{\text {op }}$ such that

$$
k\left[Q \times Q^{\mathrm{op}}\right] \otimes_{k[(,, 1) \Delta Q]} k \mid k\left[Q \times Q^{\mathrm{op}}\right] \otimes_{k\left[Q \times Q^{\mathrm{op} \cap}(a, b)(x, 1) \Delta\left(x^{-1} D \cap D\right)\right]} k .
$$

By Green's indecomposablity theorem we may assume that

$$
{ }^{(g, 1)} \Delta Q=Q \times Q^{\mathrm{op}} \cap{ }^{(a, b)(x, 1)} \Delta\left({ }^{x^{-1}} D \cap D\right) .
$$

Then we have that

$$
\left\{\left({ }^{g} u, u^{-1}\right) \mid u \in Q\right\}=\left\{\left({ }^{a x} v,{ }^{b} v^{-1}\right) \in Q \times Q^{\text {op }} \mid v \in{ }^{x^{-1}} D \cap D\right\} .
$$

Therefore, for an arbitrary element $u \in Q$ there exists an element $v \in{ }^{x^{-1}} D \cap D$ such that

$$
{ }^{g} u={ }^{a x} v, \quad u^{-1}={ }^{b} v^{-1} .
$$

Here the right-hand side of the second equation above is in $Q^{\text {op }}$ so that this is in fact $b^{-1} v^{-1} b$ in $Q$. Hence we have that

$$
{ }^{g} u={ }^{a x b} u \quad \forall u \in Q .
$$

Namely, there exists an element $y \in C_{G}(Q)$ such that $g=a x b y$. Note that ${ }^{b} Q \leqslant$ $x^{-1} D \cap D$

Since we are assuming that $\delta_{D} \zeta \in H H^{*}(k D)$ is $i k G i$-stable and the $(k D, k D)$ bimodule $k[D x D]$ is isomorphic with a direct summand of $i k G i$, we see that $\operatorname{res}_{D \cap{ }^{x} D} \zeta=$ $\operatorname{res}_{D \cap x}{ }^{x} \zeta$

$={ }^{x} \operatorname{res}_{x^{-1} D \cap D} \zeta$. Hence we obtain that

$$
\begin{aligned}
& \operatorname{res}_{Q}{ }^{g} \zeta={ }^{g} \operatorname{res}_{Q} \zeta={ }^{a x b y} \operatorname{res}_{Q} \zeta \\
& ={ }^{a x b} \operatorname{res}_{Q} \zeta \quad\left(\because y \in C_{G}(Q)\right) \\
& ={ }^{a x} \operatorname{res}_{{ }}{ }^{b} \zeta={ }^{a x} \operatorname{res}_{{ }^{b}} \zeta \quad(\because b \in D) \\
& ={ }^{a} \mathrm{res}_{x b}{ }^{x} \zeta \\
& ={ }^{a} \text { res }_{x b} \operatorname{res}_{D \cap x D}{ }^{x} \zeta \quad\left(\because{ }^{x b} Q \leqslant D \cap{ }^{x} D\right) \\
& ={ }^{a} \operatorname{res}_{{ }_{x} b} \operatorname{res}_{D \cap x} \zeta \quad(\because \text { the stability condition }) \\
& ={ }^{a} \text { res }{ }^{x} Q \zeta \\
& =\operatorname{res}_{a \times b} a_{\zeta} \\
& =\operatorname{res}{ }_{g} \zeta \\
& =\operatorname{res}_{Q} \zeta \text {. } \\
& (\because a \in D)
\end{aligned}
$$

Thus, the stability condition (S) holds. 
To prove Theorem 2 we recall some properties of the $(B, C)$-bimodule $L=L(B, C)$, which we investigated in Sasaki [6].

Theorem 4 1. The relatively projective elements $\pi_{L} \in Z(B)$ and $\pi_{L^{*}} \in Z(C)$ are both invertible.

2. We have

$$
L^{*} \otimes_{B} X \simeq Y \oplus O\left(\mathscr{Y}\left(G \times D^{\mathrm{op}}, \Delta D, H \times D^{\mathrm{op}}\right)\right) .
$$

3. We have

$$
L \otimes_{k H} Y \simeq X \oplus Z
$$

and $Z$ is relatively $\mathscr{X}\left(G \times D^{\mathrm{op}}, \Delta D, H \times D^{\mathrm{op}}\right)$-projective and every indecomposable direct summand has a trivial source.

4. If $D \triangleleft H$, then $L \otimes_{k H} Y \simeq X$.

5. The bimodule $L$ is isomorphic with a direct summand of $X \otimes_{k D} Y^{*}$.

6. Take a Sylow $C$-subpair $\left(D, b_{D}\right)$ such that $b_{D} Y(D) \neq 0$. Then $\left(D, b_{D}\right)$ is also a Sylow $B$-subpair and $b_{D} X(D) \neq 0$. The following is commutative:

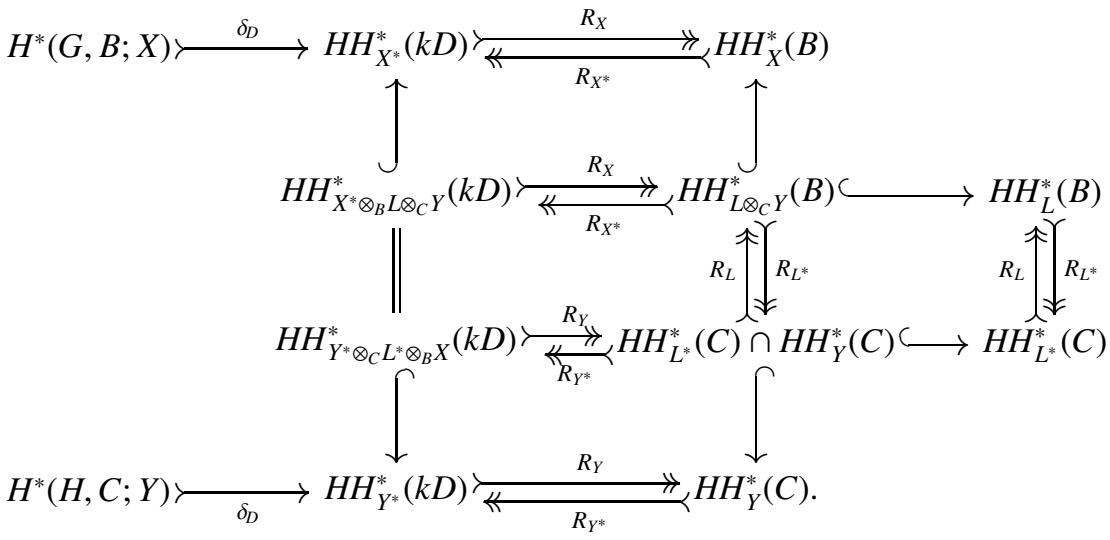

Proof of Theorem 2 For $\zeta \in H^{*}(G, B ; X)$ we see that $\left(\delta_{D} \zeta, \delta_{D} \zeta\right) \in H H^{*}(k D) \oplus H H^{*}(k D)$ is $X^{*} \otimes_{B} X$-stable; for $\eta \in H^{*}(H, C ; Y)$ we see that $\left(\delta_{D} \eta, \delta_{D} \eta\right)$ is $Y^{*} \otimes_{C} Y$-stable. Thus, if $H^{*}(G, B ; X) \subseteq H^{*}(H, C ; Y)$, then we see for $\zeta \in H^{*}(G, B ; X)$ that $\left(\delta_{D} \zeta, \delta_{D} \zeta\right)$ is $X^{*} \otimes_{B} X$ stable and $Y^{*} \otimes_{C} Y$-stable, implying that $\left(\delta_{D} \zeta, \delta_{D} \zeta\right)$ is $\left(X^{*} \otimes_{B} X\right) \otimes_{k D}\left(Y^{*} \otimes_{C} Y\right)$-stable by [2, Lemma 2.1 (a)]. We see from Theorem 4 (5) that $X^{*} \otimes_{B} L \otimes_{C} Y$ is a direct summand of $\left(X^{*} \otimes_{B} X\right) \otimes_{k D}\left(Y^{*} \otimes_{C} Y\right)$. Therefore, we see for $\zeta \in H^{*}(G, B ; X)$ that $\delta_{D} \zeta \in H H^{*}(k D)$ is $X^{*} \otimes_{B} L \otimes_{C} Y$-stable.

Suppose conversely that $\delta_{D} H^{*}(G, B ; X) \subseteq H H_{X^{*} \otimes_{B} L \otimes_{C} Y}(k D)$. Then the commutative diagram in Theorem 4 implies for $\zeta \in H^{*}(G, B ; X)$ that $\delta_{D} \zeta \in H H^{*}(k D)$ is $Y^{*}$-stable. Thus Theorem 1 implies that $\zeta \in H^{*}(H, C ; Y)$, namely we have that $H^{*}(G, B ; X) \subseteq H^{*}(H, C ; Y)$.

When the condition holds, the commutative diagram in Theorem 4 gives rise to the commutative diagram required. 


\section{Transfer map}

Lemma 5 The following conditions are equivalent.

1. $H^{*}(G, B ; X)=t H^{*}(D, k)$.

2. $\delta_{D} t H^{*}(D, k) \subseteq H H_{i k G i}^{*}(k D)$.

3. For an arbitrary element $\zeta \in H^{*}(D, k)$ and an arbitrary direct summand $k[D x D]$ of $i k G i$ the following equation holds:

$$
t_{\left(D \cap x_{D} i k G i\right)}\left(\delta_{D} \zeta\right)=t_{\left(D \cap x_{D} k[x D] \otimes_{k D} i k G i\right)}\left(\delta_{D} \zeta\right) .
$$

Proof Suppose that $H^{*}(G, B ; X)=t H^{*}(D, k)$. Then we see by Linckelmann [5, Theorem 5.6 (ii)] that

$$
\delta_{D} t H^{*}(D, k)=\delta_{D}\left(H^{*}(G, B ; X)\right) \subset H H_{i k G i}^{*}(k D) .
$$

Suppose conversely that $\delta_{D} t H^{*}(D, k) \subset H H_{i k G i}^{*}(k D)$. Then we see by Theorem 1 that $t H^{*}(D, k) \subset H^{*}(G, B ; X)$. As we have already seen for $\zeta \in H^{*}(G, B ; X)$ in Remark 1 in Introduction that $t(\zeta)=\pi_{i k G i} \zeta$, we have the following commutative diagram:

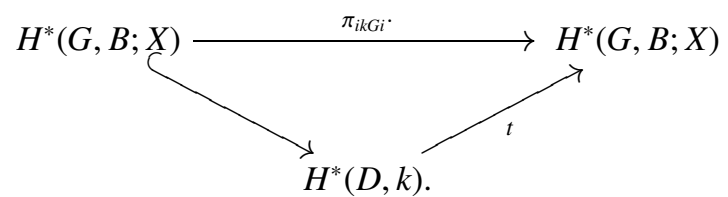

Because $\pi_{i k G i}$ is not zero, the horizontal map above is isomorphic, hence it follows that $H^{*}(G, B ; X)=t H^{*}(D, k)$.

Next, take an element $\zeta \in H^{*}(D, k)$. Then the diagonal embedding $\delta_{D} t(\zeta) \in$ $H H^{*}(k D)$ is $i k G i$-stable if and only if for an arbitrary direct summand $k[D x D]$ of $i k G i$, the element $\delta_{D} t(\zeta) \in H H^{*}(k D)$ is $k[D x D]$-stable, which is equivalent to

$$
t_{\left(D \cap x_{D} k D\right)}\left(\delta_{D} t(\zeta)\right)=t_{\left(D \cap x_{D} k[x D]\right)}\left(\delta_{D} t(\zeta)\right)
$$

Moreover, because $\delta_{D} t(\zeta)=t_{i k G i}\left(\delta_{D} \zeta\right)$, the condition above is equivalent with

$$
t_{\left(D \cap x_{D} k D\right)}\left(t_{i k G i}\left(\delta_{D} \zeta\right)\right)=t_{\left(D \cap x_{D} k[x D]\right)}\left(t_{i k G i}\left(\delta_{D} \zeta\right)\right)
$$

as desired.

In the rest of this note we would like to show some examples: when the defect group is normal and when the defect group is abelian. The referee pointed out that in these cases the $(k D, k D)$-bimodule $i k G i$ is just the module induced by the biset constructed in Broto, Levi and Oliver [1], which we mentioned in Introduction. We however would like to give direct descriptions to these facts.

Example 1 If the defect group $D$ is normal in $G$, then Conjecture holds. 
Proof Since the the defect group $D$ is normal in $G$, we have that

$$
H^{*}(G, B ; X)=H^{*}(D, k)^{N_{G}\left(D, b_{D}\right)} .
$$

The inertia index $\left|N_{G}\left(D, b_{D}\right): D C_{G}(D)\right|$ is not divisible by the prime $p$ so that

$$
H^{*}(D, k)^{N_{G}\left(D, b_{D}\right)}=\operatorname{tr}_{D C_{G}(D)}^{N_{G}\left(D, b_{D}\right)} H^{*}(D, k) .
$$

Because $D$ is normal in $G$, the $(k D, k D)$-bimodule $i k G i$ decomposes as follows:

$$
i k G i \simeq \bigoplus_{g D C_{G}(D) \in N_{G}\left(D, b_{D}\right) / D C_{G}(D)} k[D g] .
$$

Therefore we see for $\zeta \in H^{*}(D, k)$ that

$$
t(\zeta)=\sum_{g D C_{G}(D) \in N_{G}\left(D, b_{D}\right) / D C_{G}(D)} g_{\zeta}=\operatorname{tr}_{D C_{G}(D)}^{N_{G}\left(D, b_{D}\right)}(\zeta) .
$$

Example 2 Assume that the defect group $D$ is abelian. Then it follows for an arbitrary direct summand $k[D x D]$ of $i k G i$ that, as $\left(k\left[D \cap{ }^{x} D\right], k D\right)$-bimodules,

$$
k[x D] \otimes_{k D} i k G i \simeq i k G i
$$

so that the condition (3) in Lemma 5 holds.

Proof First of all we note that the $\left(k\left[{ }^{x} D\right], k D\right)$-bimodule $k[x D] \otimes_{k D} i k G i$ can be written as $x \otimes i k G i=\{x \otimes \omega \mid \omega \in i k G i\}$; the conjugate ${ }^{x} D$ acts on the left in the following manner:

$$
{ }^{x} a(x \otimes \omega)=x \otimes a \omega \quad \text { for } a \in D, \omega \in i k G i .
$$

Külshammer, Okuyama and Watanabe [3, Proposition 5] says for an arbitrary direct summand $k[D x D]$ of $i k G i$ that

$$
x^{-1}\left(D \cap{ }^{x} D, b_{D \cap{ }^{x} D}\right) \leqslant\left(D, b_{D}\right) .
$$

Since $D$ is abelian, we see that $x^{-1} \in N_{G}\left(D, b_{D}\right) C_{G}\left(D \cap{ }^{x} D\right)$; hence there exist an element $z \in N_{G}\left(D, b_{D}\right)$ and an element $c \in C_{G}\left(D \cap{ }^{x} D\right)$ such that $x^{-1}=z c$. Rewriting $z^{-1}$ as $z$ and $c^{-1}$ as $c$, we see that there exist an element $z \in N_{G}\left(D, b_{D}\right)$ and an element $c \in C_{G}\left(D \cap{ }^{x} D\right)$ such that

$$
x=c z \text {. }
$$

The action of $z \in N_{G}\left(D, b_{D}\right)$ on $i$ is given by a unit $u \in U\left(B^{D}\right)$ as follows:

$$
z i z^{-1}=u i u^{-1} \text {. }
$$

Then we have that

$$
x i x^{-1}=c z i z^{-1} c^{-1}=c u i u^{-1} c^{-1} .
$$

We would like to show that the map

$$
\begin{aligned}
c z \otimes i k G i & \rightarrow c u \otimes i k G i ; \\
c z \otimes a & \mapsto c u \otimes u^{-1} z a \quad(a \in i k G i)
\end{aligned}
$$


is a $\left(k\left[D \cap{ }^{x} D\right], k D\right)$-isomorphism.

It is enough to show that this map is a $k\left[D \cap{ }^{x} D\right]$-homomorphism. We see for $d \in D \cap{ }^{x} D$ that

$$
\begin{aligned}
d(c z \otimes a) & =d c z \otimes a=c z z^{-1} d z \otimes a=c z \otimes z^{-1} d z a \\
& \mapsto c u \otimes u^{-1} z\left(z^{-1} d z a\right)=c u \otimes u^{-1} d z a \\
& =c u \otimes d u^{-1} z a \quad\left(\because u \in U\left(B^{D}\right)\right) .
\end{aligned}
$$

On the other hand we obtain that

$$
\begin{aligned}
d\left(c u \otimes u^{-1} z a\right) & =d c \otimes u^{-1} z a & & \\
& =c d u \otimes u^{-1} z a & & \left(\because c \in C_{G}\left(D \cap{ }^{x} D\right)\right) \\
& =c u d \otimes u^{-1} z a & & \left(\because u \in U\left(B^{D}\right)\right) \\
& =c u \otimes d u^{-1} z a . & &
\end{aligned}
$$

Thus we obtain a $\left(k\left[D \cap{ }^{x} D\right], k D\right)$-isomorphism

$$
x \otimes i k G i=c z \otimes i k G i \simeq c u \otimes i k G i \simeq i k G i .
$$

We end this note with a remark on principal blocks.

Remark 2 Let $P$ be a Sylow $p$-subgroup of $G$ and let $B_{0}$ be the principal block; let $X=k G i_{0}$ be a source module. Let $M$ denote the $(k G, k P)$-bimodule $k G$; the $(k G, k P)$ bimodule $X$ is a direct summand of $M$. Then we have the following commutative diagram

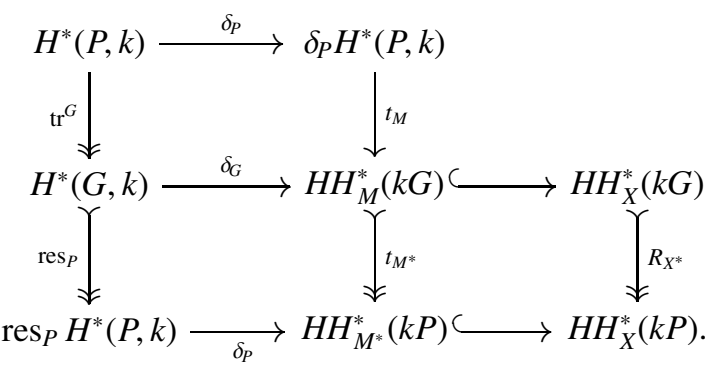

Namely we see for an element $\zeta \in H^{*}(P, k)$ that

$$
\begin{aligned}
\delta_{P} \operatorname{res}_{P} \operatorname{tr}^{G} \zeta & =t_{M^{*}} t_{M} \delta_{P} \zeta \\
& =R_{X^{*}} t_{M} \delta_{P} \zeta \\
& =\frac{1}{\pi_{X^{*}}} t_{X^{*}} t_{M} \delta_{P} \zeta \\
& =\frac{1}{\pi_{X^{*}}} t_{X^{*}{ }_{k P}} \delta_{P} \zeta \\
& =t_{X^{*}{ }_{k P}} \delta_{P}\left(\frac{1}{\pi_{X^{*}}} \zeta\right) .
\end{aligned}
$$


Thus if we define a linear map

$$
t^{\prime}: H^{*}(P, k) \rightarrow H^{*}(P, k) ; \zeta \mapsto \sum_{X^{*} \otimes_{B_{0}} M \simeq \bigoplus k[P g P]} \operatorname{tr}^{P} \operatorname{res}_{P \cap s P}{ }^{g} \zeta,
$$

then we obtain that

$$
H^{*}\left(G, B_{0} ; X\right)=\operatorname{Im} t^{\prime} .
$$

However, needless to say, this is far from our Conjecture.

\section{Acknowledgement}

The author would like to express his sincere thanks to the referee for his valuable suggestions and comments.

\section{References}

1. Broto, C., Levi, R., Oliver, B.: The homotopy theory of fusion systems. J. Amer. Math. Soc. 16, 779856 (2003)

2. Kawai, H., Sasaki, H.: Cohomology algebras of blocks of finite groups and Brauer correspondence. Algebr. Represent. Theory 9(5), 497-511 (2006)

3. Külshammer, B., Okuyama, T., Watanabe, A.: A lifting theorem with applications to blocks and source algebras. J. Algebra 232, 299-309 (2000)

4. Linckelmann, M.: On drived equivalences and local structure of blocks of finite groups. Turkish J. Math. 22, 93-107 (1988)

5. Linckelmann, M.: Transfer in Hochschild cohomology of blocks of finite groups. Algebr. Represent. Theory 2, 107-135 (1999)

6. Sasaki, H.: Cohomology algebras of blocks of finite groups and Brauer correspondence II. Algebr. Represent. Theory 13, 445-465 (2010) 